\title{
Modeling of geometrical stability of the diffraction lens mount for a promising project of the outer space observation satellite
}

\author{
G.P. Anshakov ${ }^{1}$, V.V. Salmin ${ }^{1}$, K.V. Peresypkin ${ }^{1}$, A.S. Chetverikov ${ }^{1}$, I.S. Tkachenko ${ }^{1}$

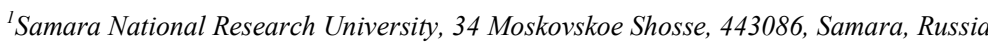

\begin{abstract}
The article gives an overview of a promising project of an observation spacecraft fitted with a diffractive optical system. The problem of ensuring a stable position of the elements of the optical system is considered. For this purpose, the load-bearing scheme of the mount of a diffraction lens previously proposed by the authors is used. In this work, a study is made of the influence of the geometric parameters of the structure on its stiffness characteristics. With the help of numerical optimization, the optimal values of the design parameters for minimizing structural mass are calculated.
\end{abstract}

Keywords: diffraction optics, space membrane optical system, finite element simulation, natural oscillations, numerical optimization

\section{Introduction}

In recent years, the project of an observation satellite that uses a diffractive lens to focus the light flux instead of a conventional mirror [1] is actively discussed in the scientific community. Due to the fact that the Fresnel lens is a thin perforated membrane, its weight is much less than the weight of a conventional mirror. This creates a possibility for creating an observation satellite with optical payload with a large aperture.

The MOIRE project [2] is of particular interest. It implies creation of an observation satellite fitted with a Fresnel lens 10 meters in diameter. This lens is to be mounted 60 meters from the body of the satellite. The overview of the system is resented in figure 1 .

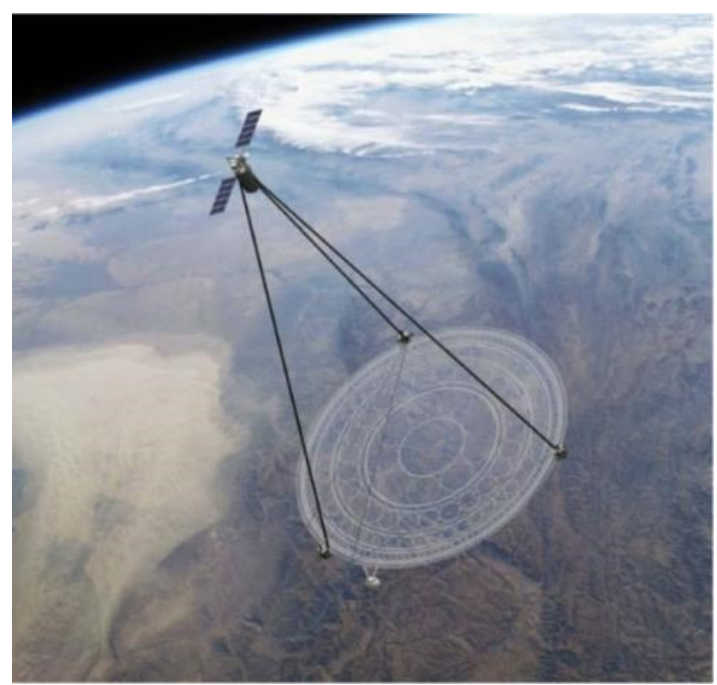

Fig. 1. MOIRE - Membrane Optical Imager for Real-Time Exploitation [1-3].

\section{Structural requirements of the diffraction lens mount}

Elements of the optical system are to be precisely positioned against each other coaxially and exactly on the right distance. This means that the dimensional stability is critical for the lens mount. Obviously, this design should be folded in the process of orbital injection, and be unfurled in orbit into the operating state. Ensuring that a structure this big remains dimensionally stable is a complex engineering task. Dimensional stability of the structure might be compromised by numerous factors: plastic deformations induced during the launch, temperature deformations, structural oscillations. The first two factors could be avoided by correct selection of the mount's material. Structural oscillations, however, will be inevitably induced during the process of positioning the telescope for image capturing. These oscillations in such a large-sized system can change the position of the lens relative to other elements of the optical system. If the amplitude of these oscillations is sufficiently large to distort the image and the decay time of these oscillations is high, it will severely hinder the image-capturing capabilities of the system. To prevent the occurrence of long-term oscillations of a lens with a large amplitude, the structure must have a sufficiently high rigidity. We propose to formulate rigidity requirements in the form of a restriction on the values of the natural vibration frequencies of the structure. Traditional observation satellites usually are equipped with solar arrays that have natural vibration frequencies in the range from $1 \mathrm{~Hz}$ to $2.5 \mathrm{~Hz}$. We have to match these values for our proposed lens mount. 


\section{Load-bearing structure of the diffractive lens mount}

In the previous paper [4] we have proposed a load-bearing scheme that could meet the aforementioned structural requirements for the mount. High rigidity in this scheme is achieved by combining three trusses into one structure by means of cables stretched between them. Strained cables load the trusses with transverse forces, which can lead to large deformations. To avoid this, in the proposed load-bearing scheme the trusses are arc-shaped and their ends are connected by a longitudinal cable as shown in figures 2-4. Such trusses function as arches and are capable of accommodating transverse loads. An overview of an observation satellite with a diffractive optic payload utilizing the proposed mount structure is shown in figure 5.

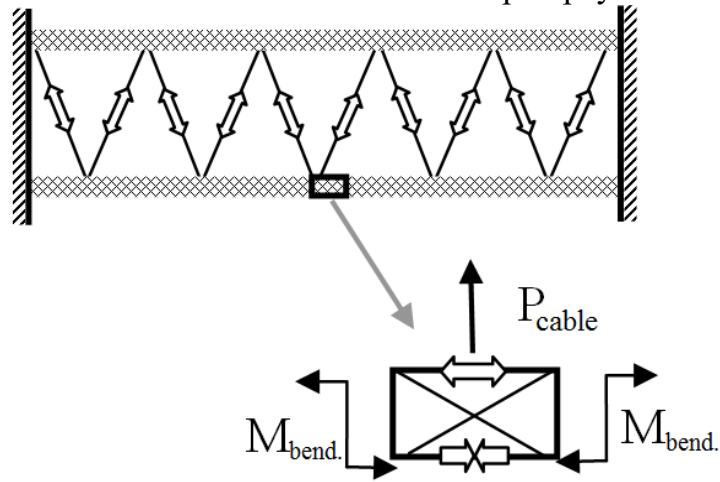

Fig. 2. Loading of straight trusses tightened with cables. Thick arrows represent internal forces induced in the structural elements and thin arrows represent external forces applied to the structure from other elements.

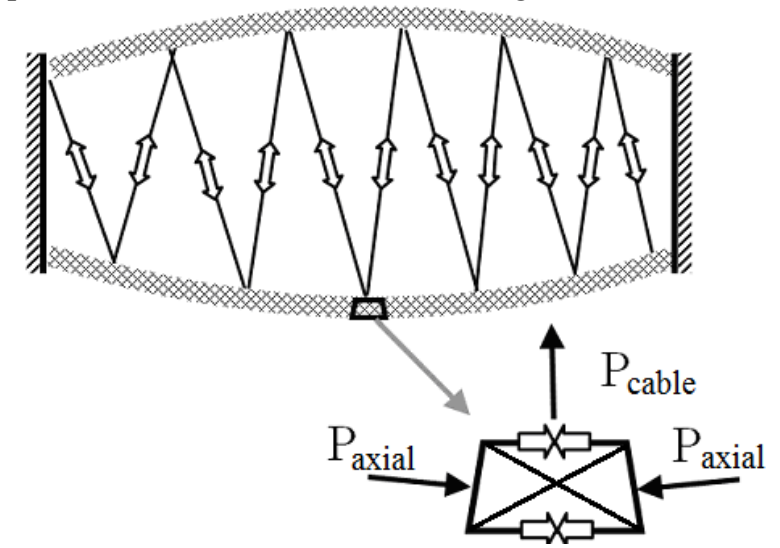

Fig. 3. Loading of arch-shaped straight trusses tightened with cables. Thick arrows represent internal forces induced in the structural elements and thin arrows represent external forces applied to the structure from other elements.

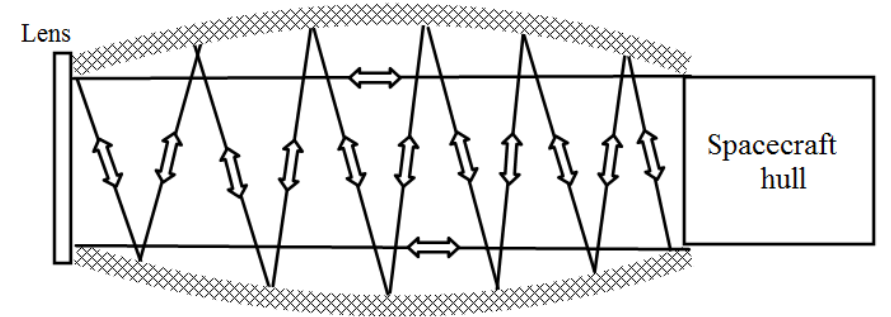

Fig. 4. Maintaining the distance between the ends of the arch-shaped trusses using longitudinal cables.

In the paper [4] we have presented a model of the loaded state of the proposed mount structure for a set of predetermined design variables and have shown the feasibility of the structural layout regarding the stiffness constraints. However, that paper did not contain a method for parametric optimization of the structure's mass. The proposed design variables are: radius of arched trusses, R; the cross-sectional area of the truss bars; the cross-sectional area of the cables. In this paper we study the influence of these parameters on the behavior of the lens mount structure.

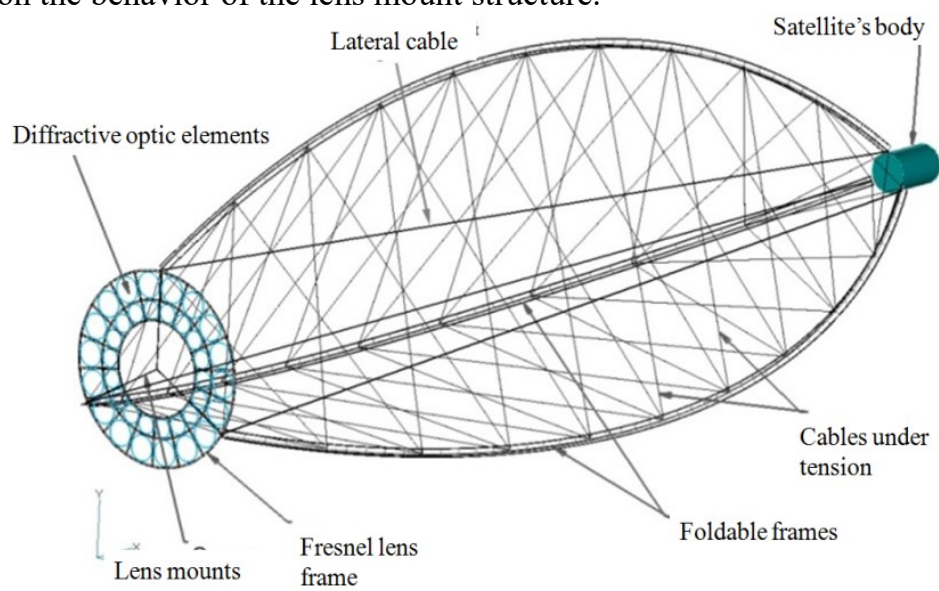

Fig. 5. An overview of an observation satellite with a diffractive optic payload utilizing the proposed mount structure.

\section{Modeling method}

Simulation of the behavior of the structure is performed in the finite element system MSC.Nastran. To estimate the rigidity of the mounting structure, the natural oscillations of a spacecraft with a diffraction lens were sought.

The search for natural oscillations in the method of finite elements consists in solving the following eigenvalue problem [2, 3]: $\left(-\omega_{i}^{2} \cdot[M]+[K]\right) \cdot\left\{U_{i}\right\}=0$, 
where $\omega_{i}-i$-th own circular frequency; $[M]$ - mass matrix; $[K]$ - stiffness matrix; $\left\{U_{i}\right\}-i$-th eigen form. The solution is performed for several lower tones of natural oscillations by the Lanczos method. The matrix of the rigidity of an elastic system within the framework of the finite element method has the following form:

$[K]=\sum_{k=1}^{N e} \int_{\text {Vek }}[B]_{k}^{T} \cdot[D]_{k} \cdot[B]_{k} d v$,

where $\mathrm{Ne}$ - number of finite elements; Vek - volume of the $k$-th finite element; $[D]_{k}$ - Hooke matrix for the material of the $k$ th finite element; $[B]_{k}$ - matrix of connection between nodal displacements and deformations: $\{\varepsilon\}_{k}=[B]_{k} \cdot\{u\}_{k} ;\{u\}_{k}$ - nodal displacements of the $k$-th finite element; $\{\varepsilon\}_{k}$ - deformations of the $k$-th finite element. Coefficients of the matrix $[B]_{k}$ could be obtained from differentiating the form function $[\Phi]_{k}$ of the finite element by the corresponding coordinates. The matrix of the masses of the elastic system in the framework of the finite element method has the following form:

$[M]=\sum_{k=1}^{N e} \rho_{k} \cdot \int_{V e k}[\Phi]_{k}^{T} \cdot[\Phi]_{k} d v$,

where $[\Phi]_{k}$ - from function of the k-th finite element: $\{u(x)\}_{k}=[\Phi]_{k} \cdot\{u\}_{k} ;\{x\}$ - coordinates of a point inside of a finite element; $\rho_{k}$ - material density of the $k$-th finite element.

\section{Influence of the radius of truss arches on the behavior of the structure}

Radii of arched trusses determine the general geometry of the structure. To determine the influence of this parameter on the natural oscillations, a number of finite element models with different values of the radius of arched trusses were built in the MSC.Nastran system (Fig. 6). Some forms of oscillations for one of the considered radii are shown in Fig. 7-10. Dependences of natural frequencies on the radius of arched trusses are shown in Fig. 11.

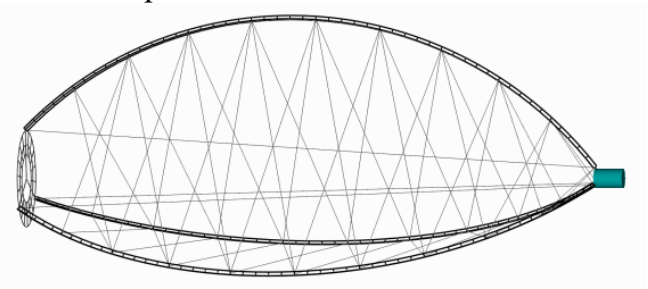

a)

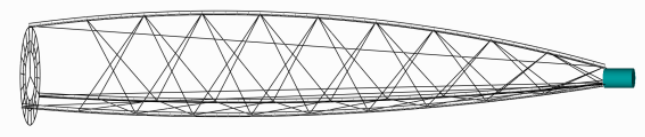

b)

Fig. 6. Finite element models of the lens mount design with different values of the radii of the arched trusses: a) $40 \mathrm{~m}$; b) $150 \mathrm{~m}$.

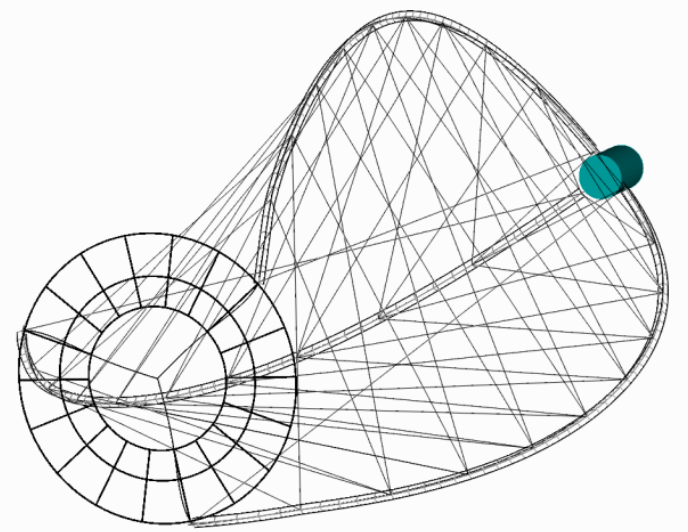

Fig. 7. Form of the first elastic tone of natural oscillations. Frequency $-0,687 \mathrm{~Hz}$. Torsion of the lens around its axis.

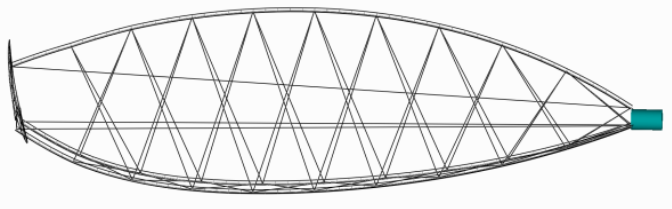

Fig. 8. Form of the second and fourth elastic tone of natural oscillations. Frequency $0,948 \mathrm{~Hz}$. The first flexural shape.

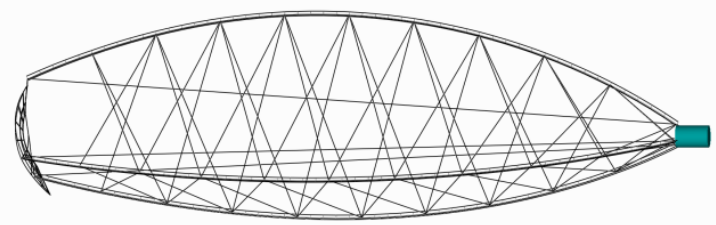

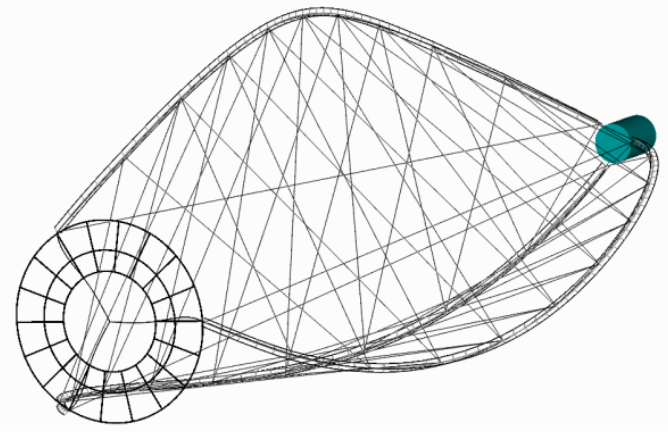

Fig. 9. Form of the fourth elastic tone of natural oscillations. Frequency - 1,14 Hz. Torsion of the lens around its axis.

Fig. 10. Form of the fifth and sixth elastic tone of natural oscillations. Frequency $-1,48 \mathrm{~Hz}$. The second flexural shape. 


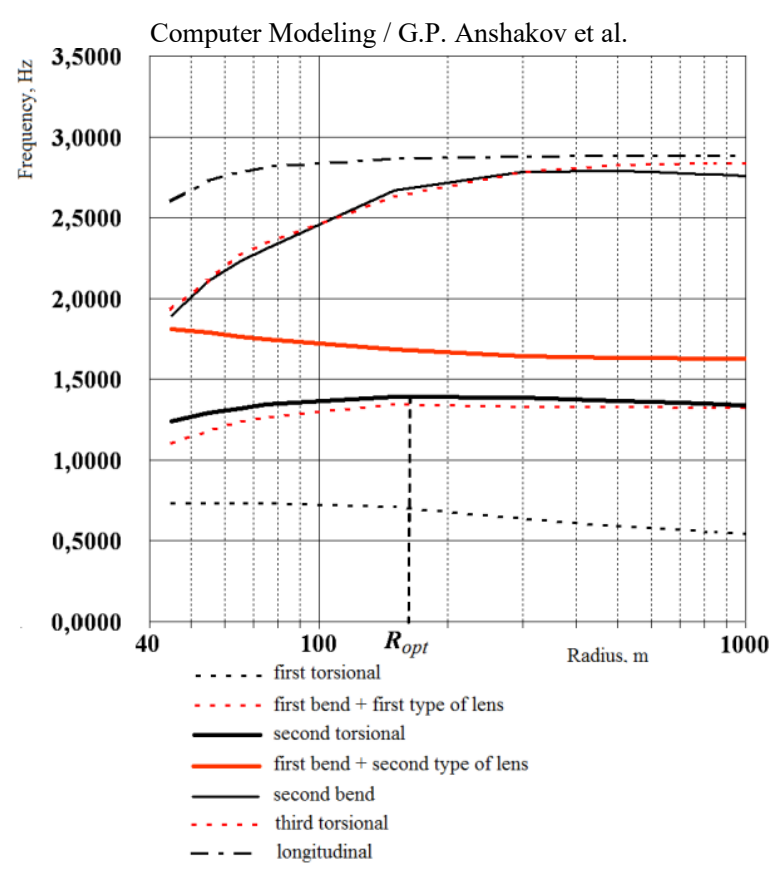

Fig. 11. Dependences of natural frequencies on the radius of arched trusses.

As far as the lower natural frequency is concerned, the smaller is the radius the higher is the stiffness. However, in the first tone of natural oscillation the lens is rotating around its optical axis. This kind of displacement does not affect the positioning of optical elements against each other and therefore does not affect the image quality of the system. The second and third forms of natural oscillations lead to tilting of the axis of the lens, which will lead to image distortion. If we would choose the radius of the arched trusses in order to maximize the frequencies of these tones, then an optimal radius value would be $150 \mathrm{~m}$.

\section{Parametric optimization of the parameters of the construction of the diffraction lens mount}

The selection of the remaining parameters of the load-bearing structure was carried out using the procedure of parametric optimization of the MSC.Nastran system. The following formulation of the optimization problem was used [5,6]:

Areas of the cross sections of the elements of the structure: the rods of the truss; beams of the lens mount, longitudinal cables and lateral cables were taken as design variables

- Design constraints: the frequencies of the five lowest tones of oscillation should not be less than $1 \mathrm{~Hz}$; the tension of the cables should not lead to buckling of the structure; inertial loads from a typical orbital rotational maneuver should not cause destruction of the material of structural elements.

- The purpose of optimization is to minimize the weight of the structure.

For parametric optimization the MSC. Nastran system uses a gradient optimization method. Figures 12 and 13 show the changes of the structure's mass during optimization, the maximum value of the constraints and the values of the design variables. The results of optimization are shown using the example of a structure with a radius of arched trusses equal to $40 \mathrm{~m}$.

As a result of optimization, the mass of the spacecraft decreased from $3620 \mathrm{~kg}$ to $3566 \mathrm{~kg}$. The value of the natural tone frequency of the lower tone increased from $0.736 \mathrm{~Hz}$ to $1.0 \mathrm{~Hz}$. Having carried out similar optimization calculations for models with different values of the radii of arched trusses, one can choose the design variant with the least mass. Thus, the optimal parameters of the load-bearing structure will be found. The authors did not perform a full series of calculations due to the preliminary and methodological nature of the study, but in the case of real design, there are no obstacles to finding optimal values of the parameters using the proposed method.

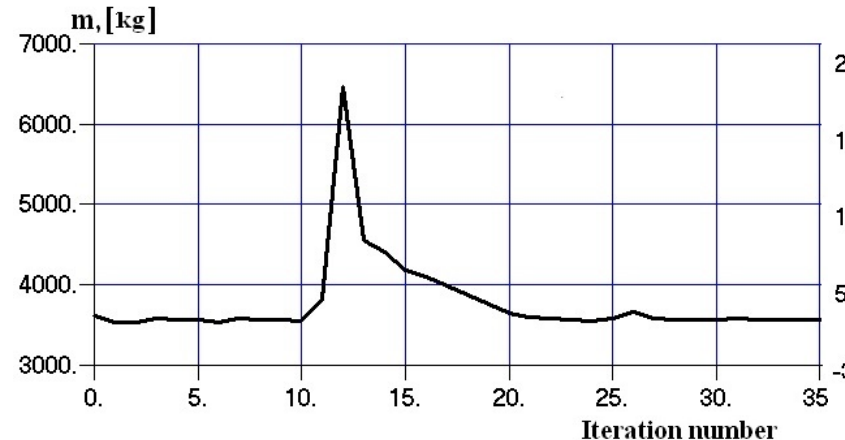

a)
Maximum value of constraints

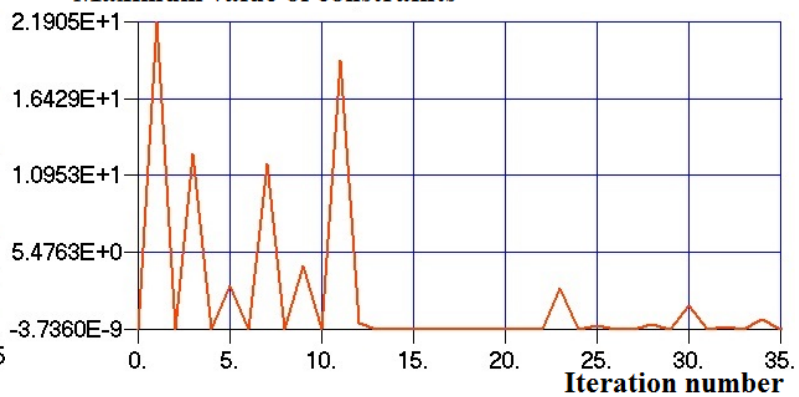

b)

Fig. 12. Change in the process of optimization: a) mass of the spacecraft (target function) and b) the maximum value of the constraints (if the value is greater than zero - the constraint is not satisfied). 


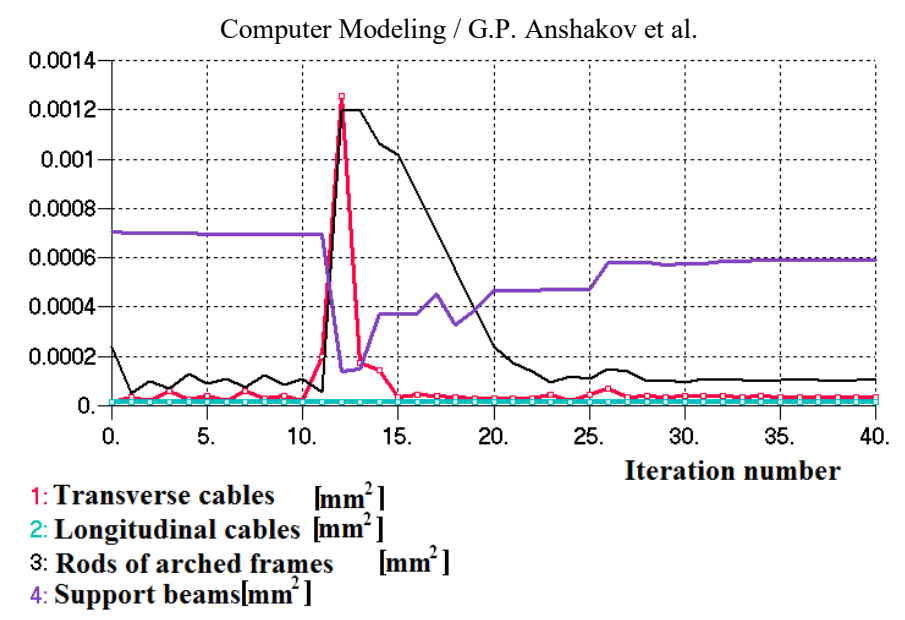

\section{Conclusion}

Fig. 13. Change of design variables during the process of optimization.

The problem of stable arrangement of the optical elements for an observation satellite with a diffractive optic payload was considered. Particular attention was given to modeling and shaping the appearance of the diffraction lens mounting structure. To solve this problem, the authors proposed a large scale load-bearing structure of high rigidity. With the help of finite element modeling, the effect of geometric parameters of a structure on its stiffness characteristics is studied. The optimal values of the design parameters ensuring minimal mass of the structure were found using numerical optimization.

\section{Acknowledgements}

This work is supported by the Ministry of Education and Science of the Russian Federation in the framework of The Federal purpose-oriented program "Research and development on priority directions of development of scientific-technological complex of Russia for 2014-2020" (agreement № 14.578.21.0229, unique identificator of the project RFMEFI57817X0229).

\section{References}

[1] Early J, Hyde R, Baron R. Twenty meter space telescope based on diffractive Fresnel lens. Proceedings of SPIE - The International Society for Optical Engineering 2004; 5166: 148-156.

[2] Atcheson P, Stewart C, Domber J, Whiteaker K, Cole J, Spuhler P, Seltzer A, Smith L. MOIRE - Initial demonstration of a transmissive diffractive membrane optic for large lightweight optical telescopes. Proceedings of SPIE - The International Society for Optical Engineering 2012; 8442 : 844221

[3] Atcheson P, Domber J, Whiteaker K, Britten JA, Dixit SN, Farmer B. MOIRE - Ground demonstration of a large aperture diffractive transmissive telescope. Proceedings of SPIE - The International Society for Optical Engineering 2014; 9143: 91431W.

[4] Salmin VV, Chtverikov A, Peresypkin KV, Tkachenko IS. Modeling control of a large-size structure in the geostationary orbit. International Conference Information Technology and Nanotechnology. Session Mathematical Modeling. CEUR Workshop Proceedings 2017; 1904: 168-173. DOI: 10.18287/16130073-2017-1904-168-173.

[5] Zienkiewicz O, Morgan K. Finite elements and approxiamtions. Moscow: Mir, 1986; 318 p. [in Russian]

[6] Zienkiewicz OC, Taylor R. The finite element method. Fifth edition. Butterwoth-Heinemann, 2000. 